\title{
Adjustment of QT dispersion assessed from 12 lead electrocardiograms for different numbers of analysed electrocardiographic leads: comparison of stability of different methods
}

\author{
Katerina Hnatkova, Marek Malik, Josef Kautzner, Yi Gang, A John Camm
}

\begin{abstract}
Objective-Normal electrocardiographic recordings were analysed to establish the influence of measurement of different numbers of electrocardiographic leads on the results of different formulas expressing QT dispersion and the effects of adjustment of QT dispersion obtained from a subset of an electrocardiogram to approximate to the true QT dispersion obtained from a complete electrocardiogram.
\end{abstract}

Subjects and methods-Resting 12 lead electrocardiograms of 27 healthy people were investigated. In each lead, the QT interval was measured with a digitising board and QT dispersion was evaluated by three formulas: (A) the difference between the longest and the shortest QT interval among all leads; (B) the difference between the second longest and the second shortest QT interval; (C) SD of QT intervals in different leads. For each formula, the "true" dispersion was assessed from all measurable leads and then different combinations of leads were omitted. The mean relative differences between the QT dispersion with a given number of omitted leads and the "true" QT dispersion (mean relative errors) and the coefficients of variance of the results of QT dispersion obtained when omitting combinations of leads were compared for the different formulas. The procedure was repeated with an adjustment of each formula dividing its results by the square root of the number of measured leads. The same approach was used for the measurement of QT dispersion from the chest leads including a fourth formula (D) the SD of interlead differences weighted according to the distances between leads. For different formulas, the mean relative errors caused by omitting individual electrocardiographic leads were also assessed and the importance of individual leads for correct measurement of QT dispersion was investigated.

Results-The study found important differences between different formulas for assessment of QT dispersion with respect to compensation for missing measurements of QT interval. The standard max-min formula (A) performed poorly (mean relative errors of $6 \cdot 1 \%$ to $18 \cdot 5 \%$ for missing one to four leads) but was appropriately adjusted with the factor of $1 / \sqrt{ } n$ ( $n=$ number of measured leads). In a population of healthy people such an adjustment removed the systematic bias introduced by missing leads of the 12 lead electrocardiogram and significantly reduced the mean relative errors caused by the omission of several leads. The unadjusted SD was the optimum formula (C) for the analysis of 12 lead electrocardiograms, and the weighted standard deviation (D) was the optimum for the analysis of six lead chest electrocardiograms. The coefficients of variance of measurements of QT dispersion with different missing leads were very large (about 3 to 7 for one to four missing leads). Independently of the formula for measurement of QT dispersion, omission of different leads produced substantially different relative errors. In 12 lead electrocardiograms the largest relative errors $(>10 \%)$ were caused by omitting lead aVL or lead V1.

Conclusions-Because of the large coefficients of variance, the concept of adjusting the QT dispersion for different numbers of electrocardiographic leads used in its assessment is difficult if not impossible to fulfil. Thus it is likely to be more appropriate to assess QT dispersion from standardised constant sets of electrocardiographic leads.

(Br Heart f 1994;72:390-396)

Different electrophysiological mechanisms are likely to contribute to arrhythmogenesis and to the pathophysiology of sudden cardiac death. As our understanding of these mechanisms increases, new methods for their assessment are solicited.

Recently, several groups have studied the possibility of assessing the dispersion of duration of QT interval from the standard 12 lead electrocardiogram. ${ }^{1-4}$ The concept of measurement of QT dispersion is based on the idea that increased differences in durations of QT intervals in individual electrocardiographic leads reflect, among other things, 
increased inhomogeneity in myocardial repolarisation, which is an arrhythmogenic factor. ${ }^{56}$

Although this concept is attractive, several technical factors complicate the measurement of QT dispersion. The definition of the end of the $T$ wave varies from study to study, especially in cases with pronounced $U$ waves. ${ }^{17}$ Similarly, an optimum formula for expressing the QT dispersion has not been established, and different formulas have been selected empirically and applied in different clinical settings. The differences between the analysis of the truly simultaneous 12 lead electrocardiogram and the more common sequential records of groups of electrocardiographic leads have been acknowledged but not properly investigated.

One of the main technical limitations is the necessity of analysing different numbers of electrocardiographic leads in different recordings. Often, the end of the $T$ wave is not clearly distinguishable in one or more of the 12 leads. Less often, recording noise or the presence of ventricular extrasystoles exclude a certain lead from measurement of QT interval. Thus some form of adjustment of the measures of QT dispersion for the number of analysed leads has been proposed, ${ }^{2}$ although the possibilities for such an adjustment have not been systematically investigated.

This study analysed a set of electrocardiographic recordings taken from healthy volunteers to establish the influence of different numbers of measured electrocardiographic leads on the results provided by different formulas expressing QT dispersion and the optimum adjustment of QT dispersion obtained from a subset of an electrocardiogram to approximate to the true QT dispersion obtained from a complete electrocardiogram. The study was a technical and methodological attempt to form a systematic basis for the adjustment of measurement of QT dispersion for different numbers of analysed electrocardiographic leads.

\section{Subjects and methods}

SUBJECT POPULATION AND RECORDINGS

The initial population of the study consisted of 28 healthy volunteers (18 men, mean (range) age $31(22-39)$ years). None of the subjects had any cardiovascular symptoms and clinical examinations for all were normal.

Within a period of one month, four standard 12 lead electrocardiograms were made from each subject with a Hewlett-Packard Page Writer II recorder, model 4745. The recorder was set at $25 \mathrm{~mm} / \mathrm{s}$ paper speed. Each recording was subsequently analysed by two medically qualified observers who operated independently. Of each set of four electrocardiograms, one record with the highest number of leads with a measurable $T$ wave pattern was selected (judged by a retrospective agreement between both observers). In 16 subjects, an electrocardiogram with a clear $\mathrm{T}$ wave pattern in all 12 leads was obtained; in 11 subjects, an electrocardiogram with a clear $T$ wave in 11 leads was taken. In the remaining subject, fewer than 11 leads had a clear $\mathrm{T}$ wave in all four recordings and this subject was excluded from the study. Of the 27 subjects included in the study, 25 had a clear $\mathrm{T}$ wave pattern present in all six chest leads (V1-V6).

In each recording, QT intervals were measured in each lead from the onset of the QRS complex to the end of the $T$ wave. In the presence of a $U$ wave, the QT interval was measured to the nadir between $T$ and $U$ waves. A Telepad PC digitiser model 554 1121 with a resolution of $0.1 \mathrm{~mm}$ was used for the measurement. Two measurements of the QT interval were taken from each measurable lead of each electrocardiogram by both observers and were subsequently averaged and used in the study as the correct value of that particular lead.

\section{METHODS OF MEASUREMENT OF QT} DISPERSION

The analysis of QT dispersion was performed on the complete 12 lead electrocardiograms as well as selectively on six lead electrocardiograms consisting only of the chest leads. The population of 25 subjects having a complete set of data of QT intervals in the precordial leads was used for this purpose.

On the complete 12 lead electrocardiograms, three different formulas were used that expressed the dispersion numerically:

(A) the difference max-min between the longest (max) and shortest (min) QT interval among all measured leads;

(B) the difference max $^{\prime}-$ min' $^{\prime}$ between the second longest $\left(\max ^{\prime}\right)$ and second shortest $\left(\min ^{\prime}\right)$ QT interval among all measured leads,

(C) the $S D$ of the durations of QT intervals in all measured leads.

The same formulas were used for the analysis of the chest leads but a fourth method was also used that weighted the interlead differences by the distances between the leads (for example a difference between the QT interval in V5 and V6 contributed to the resulting measure of QT dispersion more than did the same difference between V1 and V6). Specifically, the formula used was:

(D)

$$
\sqrt{\sum_{i \neq j} \frac{\left(Q T_{i}-Q T_{j}\right)^{2}}{|i-j|}} / \sum_{i \neq j} \frac{1}{|i-j|}
$$

where $Q T_{\mathrm{i}}$ was the duration of the $\mathrm{QT}$ interval in the lead Vi.

Thus methods (A), (B), and (C) were applied to 12 lead electrocardiograms, whereas methods (A), (B), (C), and (D) were applied to six lead precordial electrocardiograms.

STABILITY OF THE METHODS

The stability of different methods when used to assess QT dispersion from a different number of electrocardiographic leads was investigated by omitting various leads from the assessment. A true value $\left(D_{0}\right)$ of QT dispersion was obtained from each 12 lead and six lead electrocardiogram based on the complete 
set of available leads. Then, the measurement was repeated for all combinations of leads in which one lead, two leads, three leads, etc, were missing. From all combinations of leads in which the same number $m$ of leads were missing, the mean value $D_{m}$ of the coefficient of variance $V_{m}$ (SD/mean) of the QT dispersion measurements were computed. In more detail: in electrocardiograms with all 12 measurable leads, $D_{0}$ was the value of QT dispersion taken from all leads. Then, 12 values of QT dispersion were obtained from sets of 11 leads in which the leads I, II, III, aVR, aVL, etc, were missing; $D_{1}$ was the mean of these 12 values and $V_{1}$ was their $S D / D_{1}$. Similarly, 66 values of $\mathrm{QT}$ dispersion were obtained from sets of 10 leads in which different combinations of two leads were missing; and $D_{2}$ was the mean and $V_{2}$ was the coefficient of variance of these values. In the same way, $D_{3}$ and $V_{3}$ were obtained from 220 values of QT dispersion with combinations of three leads missing; $D_{4}$ and $V_{4}$ from 495 values of QT dispersion with combinations of four leads missing, etc.

Further, the difference between the mean $D_{m}$ and the true measurement $D_{0}$ was expressed as the relative error $\left(E_{m}\right)$ of the standard measurement where $E_{m}=$ $\left(D_{m}-D_{0}\right) / D_{0}$. The measurement of QT dispersion in 12 lead electrocardiograms was performed for all combinations of one to six leads missing, whereas the measurement in six lead chest electrocardiograms was repeated for all combinations with one and two leads missing.

As well as the basic formulas, adjusted formulas were used in which the result from the basic formula was divided by the square root of the number $(\sqrt{ } n)$ of electrocardiographic leads used in the measurement. ${ }^{2}$ In the text, these adjustments of the basic formulas (A), (B), (C), and (D) are denoted as (a), (b), (c), and $(\mathrm{d})$,

Table 1 Descriptive statistics of unadjusted electrocardiographic QT interval dispersion (ms) in the normal population of the study evaluated by different formulas

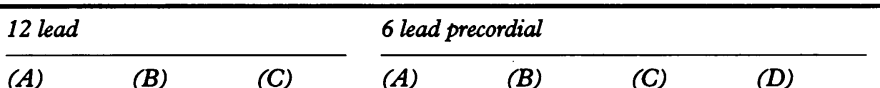

Mean (SD) 46.2 (20.8) 24.8 (10.3) $12.7(5 \cdot 4) \quad 34 \cdot 9(21 \cdot 1) \quad 11 \cdot 0(4 \cdot 8) \quad 11.9(7 \cdot 0) \quad 17 \cdot 2(10 \cdot 0)$

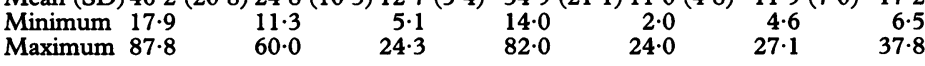

Table 2 Pearson's correlation coefficients between unadjusted individual electrocardiographic measures of $Q T$ dispersion $(n=25)$ evaluated by different formulas

\begin{tabular}{|c|c|c|c|c|c|c|c|}
\hline & \multicolumn{3}{|l|}{12 lead } & \multicolumn{4}{|c|}{6 lead precordial } \\
\hline & $(A)$ & (B) & (C) & $(A)$ & (B) & (C) & (D) \\
\hline $\begin{array}{l}12 \text { Lead } \\
\text { (A) } \\
\text { (B) } \\
\text { (C) }\end{array}$ & $\begin{array}{l}1.000 \\
0.571 \\
0.977\end{array}$ & $\begin{array}{l}1.000 \\
0.696\end{array}$ & 1.000 & & & & \\
\hline $\begin{array}{l}6 \text { Lead precordial } \\
\text { (A) } \\
\text { (B) } \\
\text { (C) } \\
\text { (D) }\end{array}$ & $\begin{array}{l}0.758 \\
0.381 \\
0.762 \\
0.762\end{array}$ & $\begin{array}{l}0.398 \\
0.461 \\
0.413 \\
0.427\end{array}$ & $\begin{array}{l}0.739 \\
0.391 \\
0.749 \\
0.748\end{array}$ & $\begin{array}{l}1.000 \\
0.252 \\
0.995 \\
0.993\end{array}$ & $\begin{array}{l}1 \cdot 000 \\
0 \cdot 298 \\
0 \cdot 318\end{array}$ & $\begin{array}{l}1.000 \\
0.995\end{array}$ & 1.000 \\
\hline
\end{tabular}

IMPORTANCE OF INDIVIDUAL LEADS

The differences between the influence of omitting different electrocardiographic leads on the resulting value of QT dispersion were investigated systematically for all leads. For each lead $L$, the value of QT dispersion assessed from all other leads-that is, with the lead $L$ missing $-D_{1}{ }^{L}$ was calculated and its difference from the true dispersion $D_{0}$ was expressed in terms of the relative error $E_{1}{ }^{L}=$ $\left(D_{1}^{L}-D_{0}\right) / D_{0}$. Similarly, the QT dispersion $D_{2}{ }^{L M}$ assessed with two leads $L$ and $M$ missing was obtained for all combinations of $L$ and $M$ and its relative error $E_{2}{ }^{L M}=\left(D_{2}{ }^{L M}-D_{0}\right) / D_{0}$ was calculated.

The relative errors $E_{1}{ }^{L}$ and $E_{2}{ }^{L M}$ obtained from the subjects of the study with different unadjusted formulas for assessment of QT dispersion were compared. In more detail, the means $(S D)$ of $E_{1}{ }^{L}$ and $E_{2}{ }^{L M}$ were calculated for 12 lead electrocardiograms from subjects in which both leads $L$ and $M$ were measurable; the means (SD) of $E_{1}{ }^{L}$ were calculated for six lead chest electrocardiograms from all subjects with the complete QT interval data from all precordial leads.

\section{STATISTICS}

The correspondence between results of $\mathrm{QT}$ dispersion obtained by applying different formulas to the 12 lead electrocardiograms and to the six lead precordial electrocardiograms was assessed by correlation coefficients. For this purpose, only the subgroup of 25 patients was used in whom both the 12 lead electrocardiograms and the six lead precordial electrocardiograms were analysed. A paired Wilcoxon sign rank test was used to test:

The differences between $D_{o}$ and $D_{m}$ for the same formula and a different number of missing leads $(m)$.

The differences between absolute values of relative errors $E_{m}$ of different formulas used with the same number of missing leads $(m)$.

The differences between the coefficients of variance $V_{m}$ of different formulas used with the same number of missing leads,

The differences between relative errors $E_{1}{ }^{L}$ and $E_{1}{ }^{M}$ for the same formula and for different combinations of missing individual leads $L$ and $M$. A $P$ value $<0.05$ was considered to be statistically significant.

A non-parametric rank test was used because of the unknown distribution of QT dispersion and its assessment errors. For the relative errors of some formulas a one tail rather than two tail test was more suitable. Specifically, because of the principles of their formulas, the results of (A) and (B) applied to incomplete electrocardiograms were smaller than the results obtained from complete electrocardiograms.

\section{Results}

VALUES OF QT DISPERSION OBTAINED FROM COMPLETE ELECTROCARDIOGRAMS

Table 1 shows the descriptive statistics of the results of individual formulas applied to electrocardiograms without artificially omitting 
any leads. Table 2 shows the correlation coefficients between the results of individual formulas. With the exception of (B), the different formulas applied to the same electrocardiograms provided very strongly correlated results. The measurements of QT dispersion in the 12 lead and corresponding six lead precordial electrocardiograms also showed reasonably high correlation-again with the exception of the method (B).

COMPLETE 12 LEAD ELECTROCARDIOGRAMS Figure 1 shows the results related to the complete 12 lead electrocardiograms. Visually (C) - that is, the $S D$ of QT interval durations in individual leads-was more stable than any other of the tested formulas. Further, we found that the adjustment factor $1 / \sqrt{ } n(n=$ number of measured leads) substantially improved the stability of methods (A) and (B) by reducing their relative errors. On the contrary, this adjustment factor was inappropriately strong for method (C). The relative errors of the overadjusted (c) were larger than those of (C) (fig 2).

It can be also seen in fig 1 that (B) was much less stable than (A). Apart from (a), all formulas resulted in a systematic bias when applied to data with missing leads. Formulas (A), (B), (C), and (b) tended to produce
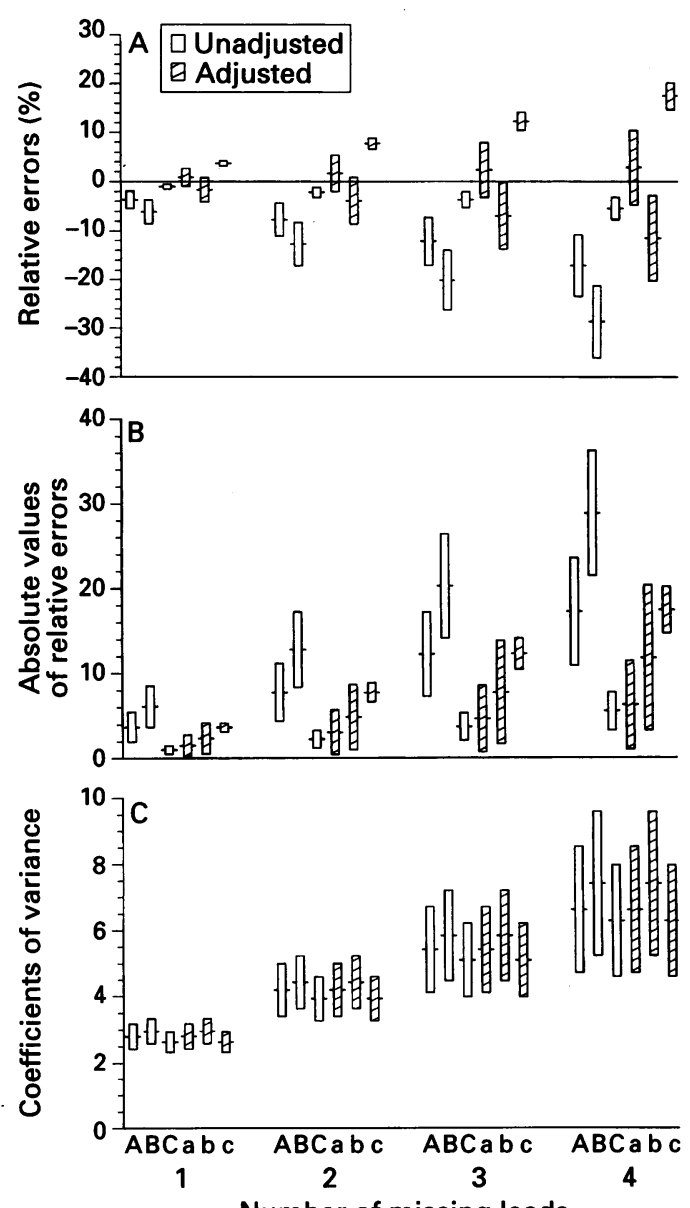

Figure 1 (A) Mean relative errors of individual methods of assessment of $Q T$ dispersion caused by omitting combinations of one, two, three, and four leads of the 12 lead electrocardiograms. (B) Mean absolute values of such relative errors, and $(C)$ coefficients of variance of the results of $Q T$ dispersion among all such combinations. Values are mean (SD).

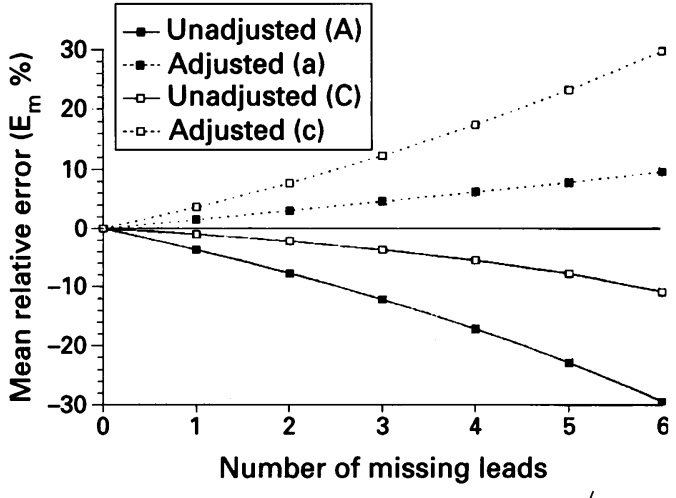

Figure 2 Performance of the adjustment factor $1 / \sqrt{ } n$ ( $n=$ number analysed leads) on methods $(A)$ and $(C)$ used with 12 lead electrocardiograms. Note the parabolic shape of the curve of relative errors of $(A)$, this relation leads to the improvement from $(A)$ to $(a)$. On the contrary, the relative errors of (C) are closer to zero and the factor $1 / \sqrt{n}$ leads to overadjustment. Even the method (a) is, on average, slightly overadjusted (possibly, a factor $1 / \sqrt[3]{n}$ might have been more appropriate to adjust the method $(A)$ in this set of data).

smaller values whereas method (c) produced larger values. Finally, we found surprisingly large values of the coefficient of variance. Independent of the method used, even the omission of different singular leads resulted in values of QT dispersion, the $S D$ of which was almost three times higher than their mean.

The visual comparisons and findings (fig 1) were confirmed by statistical analysis. For (a), the means $D_{m}$ (for $\mathrm{m}=1$ to 4 ) did not differ significantly from $D_{0}$; for all other formulas the bias was significant $(P<0.01)$. Table 3 shows the statistical differences between the absolute values of relative errors $E_{2}$ of different formulas applied to electrocardiograms with two missing leads. Exactly the same results were obtained for the comparison of relative errors $E_{1}$ and $E_{3}$ (missing one and three leads). The lowest (in absolute terms) relative error $E_{2}$ was noted for (C) and the second lowest for (a). The differences between relative errors of these formulas were not statistically significant but most of the differences between other formulas were.

As the adjustment factor $1 / \sqrt{ } n$ does not change the coefficient of variance, the same values of $V_{m}$ were obtained for (A) and (a), (B) and (b), etc. The differences in the coefficient of variance $V_{m}$ were systematically significant between $(A)$ and $(C)(P<0.05)$.

Table 3 Statistical comparisons of absolute values of relative errors of QT dispersion measurement evaluated by different formulas and caused by two missing leads in 12 lead electrocardiograms

\begin{tabular}{llll|lll}
\hline Formula & (A) & (B) & (C) & (a) & (b) & (c) \\
\hline (A) & & 0.005 & & & \\
(B) & & & & & \\
(C) & 0.005 & 0.005 & & 0.01 & 0.005 \\
\hline (a) & 0.005 & 0.005 & & & 0.005 \\
(b) & 0.005 & 0.005 & & & 0.005 \\
(c) & & 0.005 & & & \\
\hline
\end{tabular}

The table shows $P$ values (Wilcoxon paired rank signed test) of those cases in which the absolute values of the relative erro $E_{2}$ of the formula shown in the horizontal line were significantly smaller than the absolute values of the same error of the formula shown in the vertical column-for example, the errors of (A) were significantly smaller than the errors of (B). 
Figure 3 Results of assessment of $Q T$ dispersion caused by omitting combinations of one and two leads from the electrocardiograms. Values are mean $(S D)$ ). six chest lead
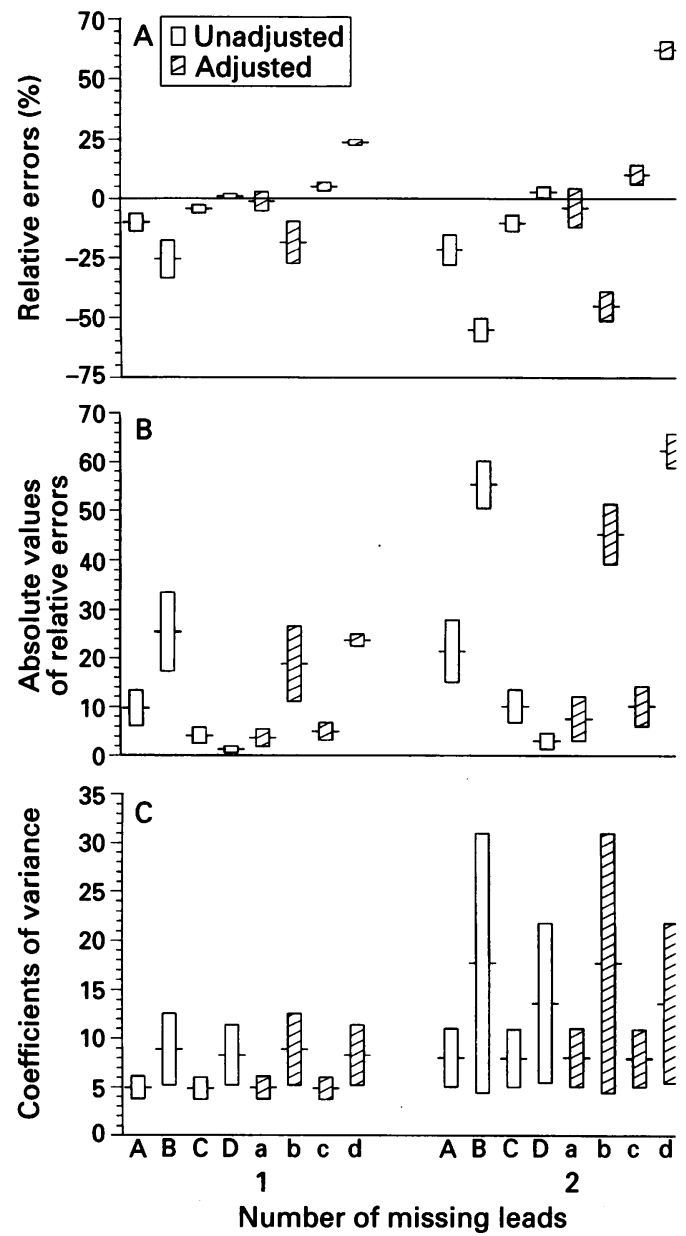

SELECTIVE MEASUREMENT OF CHEST LEADS

Figure 3 shows the results obtained with the selective analyses of six chest electrocardiographic leads. Visually we found the smallest relative errors $E_{m}$ with (D), which was followed by (a), and (C). The coefficients of variance were even larger than for the complete 12 lead electrocardiograms. Even when omitting systematically only one of all six chest leads, the mean of $V_{1}$ values in the population was $>5$. So when measuring QT dispersion from chest leads with one lead missing, the SD of six values of QT dispersions assessed with missing V1, V2, up to V6 was on average more than five times larger than their mean.

In the statistical evaluation, we found a systematic bias with all formulas. When reducing the number of chest leads (A), (B),

Table 4 Statistical comparisons of absolute values of relative errors of QT dispersion measurement evaluated by different formulas caused by one missing lead in six lead chest electrocardiograms

\begin{tabular}{lllll|llll}
\hline Formula & $(A)$ & $(B)$ & $(C)$ & $(D)$ & (a) & (b) & (c) & (d) \\
\hline (A) & & 0.005 & & & & 0.005 & & 0.005 \\
(B) & & & & & & \\
(C) & 0.005 & 0.005 & & & 0.005 & & 0.05 \\
(D) & 0.005 & 0.005 & 0.005 & & 0.005 & 0.005 & 0.005 & 0.005 \\
\hline (a) & 0.005 & 0.005 & & & & 0.005 & 0.025 & 0.005 \\
(b) & & 0.005 & & & & & & 0.005 \\
(c) & 0.005 & 0.005 & & & & 0.005 & & 0.005 \\
(d) & & & & & & & &
\end{tabular}

The table shows $P$ values (Wilcoxon paired rank signed test) of those cases in which the absolute values of the relative error $E_{1}$ of the formula shown in the horizontal line were significantly smaller than the absolute values of the same error of the formula shown in the vertical columnfor example, the errors of formula (A) were significantly smaller than the errors of formula (B)
(C), (a) and (b) tended to produce smaller values of QT dispersion whereas (D), (c) and (d) produced larger values (all $\mathrm{P}<0.01$ for both one and two leads missing). Table 4 shows statistical comparisons of the absolute values of relative errors $E_{1}$. In these terms, (D) was significantly better than any other method whereas methods (B) and (d) were significantly worse than any other method.

The statistical evaluation also confirmed the visual finding that the coefficients of variance $V_{m}$ of (A) and (C) were significantly smaller that those of (B) and (D) $(P<0.01)$.

\section{CONTRIBUTION OF INDIVIDUAL LEADS}

Figure 4 shows the distribution of QT intervals in individual electrocardiographic leads on a relative scale from the shortest to the longest QT interval. In the records from the population in this study, the shortest QT interval was most often found in leads aVL and V1, whereas the longest QT interval was most often recorded in leads V2 and V3. It is therefore not surprising that omission of one or more of these leads had a larger impact on the assessment of QT dispersion than omission of other leads. Table 5 shows the relative errors $E_{1}{ }^{L}$ of assessment of QT dispersion caused by omitting individual leads from complete 12 lead electrocardiograms. Table 6 shows the same errors for omissions of individual leads from six lead precordial electrocardiograms.

With 12 lead electrocardiograms, the largest errors were found when omitting the leads aVL or V1, which were significantly greater (in absolute terms) for (A) and (C) than the errors caused by omitting any other lead ( $P<0.02-0.0003)$. Formula (B) was, in general, more affected by omitting any individual leads than methods (A) and (C). With six lead precordial electrocardiograms, the errors caused by omitting lead V1 were also the largest but, especially with (B) and (D), were comparable with the errors caused by omitting other chest leads.

Figure 5 shows the mean relative errors $E_{2}{ }^{L M}$ of assessment of QT dispersion caused by omitting different combinations of two leads from complete 12 lead electrocardiograms. The differences between different combinations were even larger than when omitting single leads. For instance, with (A),

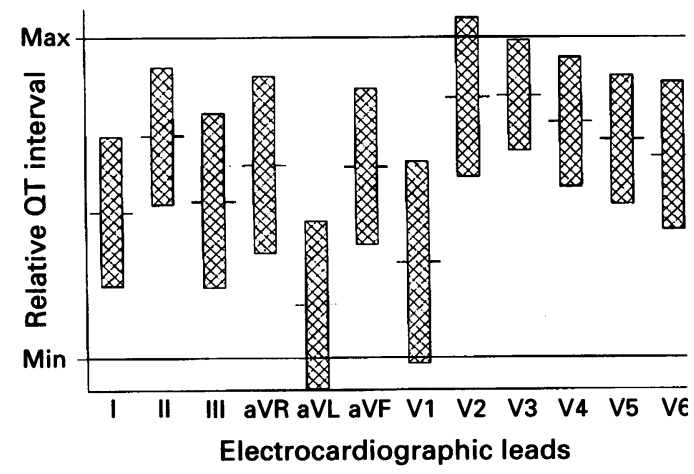

Figure 4 The $O T$ interval of each lead of each recording used in the study was expressed on a scale from the minimum to the maximum $Q T$ interval of the same electrocardiogram. Values are mean (SD)) 
Table 5 Relative errors (\%) of $Q T$ dispersion evaluated by different formulas and caused by omission of different individual leads from complete 12 lead electrocardiograms

\begin{tabular}{|c|c|c|c|}
\hline $\begin{array}{l}\text { Missing } \\
\text { lead }\end{array}$ & $\begin{array}{l}(A) \\
\text { mean }(S D)\end{array}$ & $\begin{array}{l}\text { (B) } \\
\text { mean }(S D)\end{array}$ & $\begin{array}{l}(C) \\
\text { mean }(S D)\end{array}$ \\
\hline $\begin{array}{l}\text { I } \\
\text { II } \\
\text { III } \\
\text { aVR } \\
\text { aVL } \\
\text { aVF } \\
\text { V1 } \\
\text { V2 } \\
\text { V3 } \\
\text { V4 } \\
\text { V5 } \\
\text { V6 }\end{array}$ & $\begin{array}{c}-1.51(5.36) \\
-0.33(0.91) \\
-2.27(10.67) \\
-1.56(4.62) \\
-17.02(20.19) \\
-1.39(7.11) \\
-15.79(23.29) \\
-4.86(9.06) \\
-3.71(9.49) \\
-1.05(3.18) \\
-0.27(1.39) \\
0.00(0.00)\end{array}$ & $\begin{array}{c}-8.21(12.67) \\
-2.24(5.69) \\
-7.60(13.55) \\
-5.84(13.04) \\
-17.26(11.28) \\
-5.44(12.43) \\
-13.55(12.95) \\
-6.41(9.23) \\
-5.71(9.89) \\
-1.91(4.77) \\
-2.41(7.03) \\
-1.12(4.19)\end{array}$ & $\begin{array}{r}0.74(6.05) \\
2.90(1.96) \\
-0.49(10.62) \\
0.63(6.62) \\
-15.11(16.76) \\
1.35(6.25) \\
-13.49(20.71) \\
-2.09(6.80) \\
-0.48(6.49) \\
2.12(3.14) \\
2.67(3.12) \\
3.01(2.43)\end{array}$ \\
\hline
\end{tabular}

Table 6 Mean (SD) relative errors (\%) of QT dispersion evaluated by different formulas and caused by omission of different individual leads from complete six lead precordial electrocardiograms

\begin{tabular}{|c|c|c|c|c|}
\hline $\begin{array}{l}\text { Missing } \\
\text { lead }\end{array}$ & $\begin{array}{l}(A) \\
\text { mean }(S D)\end{array}$ & $\begin{array}{l}\text { (B) } \\
\text { mean }(S D)\end{array}$ & $\begin{array}{l}(C) \\
\text { mean }(S D)\end{array}$ & $\begin{array}{l}(D) \\
\text { mean }(S D)\end{array}$ \\
\hline $\begin{array}{l}\text { V1 } \\
\text { V2 } \\
\text { V3 } \\
\text { V4 } \\
\text { V5 } \\
\text { V6 }\end{array}$ & $\begin{array}{r}-37.52(29.02) \\
-8.95(13.24) \\
-4.85(12.22) \\
-2.13(6.35) \\
-1.89(7.50) \\
-2.93(6.68)\end{array}$ & $\begin{array}{l}-36.32(28 \cdot 83) \\
-33 \cdot 71(28 \cdot 10) \\
-18 \cdot 01(25 \cdot 55) \\
-24 \cdot 22(28 \cdot 55) \\
-18 \cdot 88(29 \cdot 15) \\
-21.26(23.94)\end{array}$ & $\begin{array}{r}-33.01(29.79) \\
-4.85(13.95) \\
1.05(13.32) \\
4.33(5.83) \\
4.41(7.73) \\
3.17(8.05)\end{array}$ & $\begin{array}{r}-46.16(39.08) \\
-18.42(24.78) \\
10.07(27.57) \\
22.90(15.94) \\
24.16(19.22) \\
13.56(14.66)\end{array}$ \\
\hline
\end{tabular}
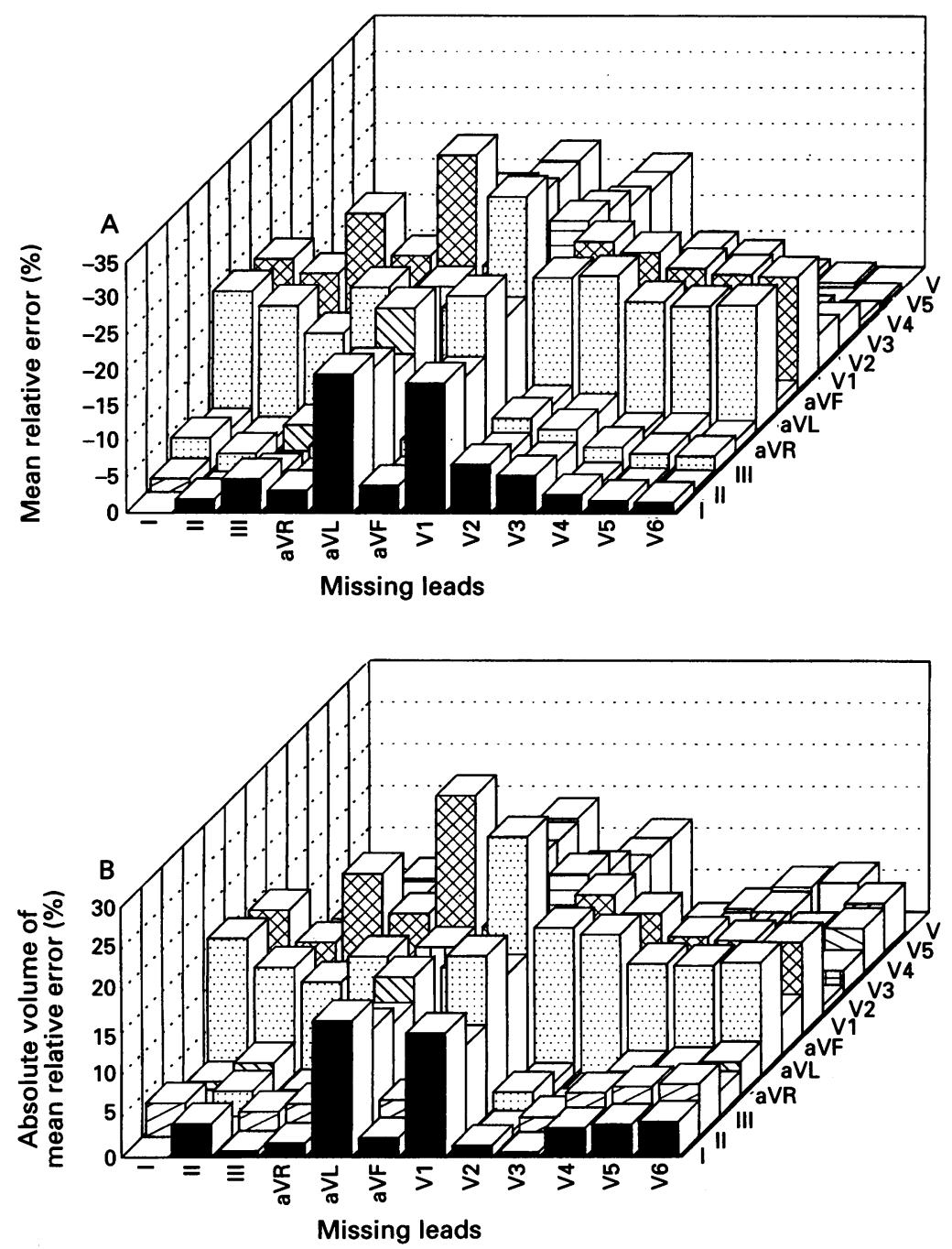

Figure 5 Relative errors $E_{2}^{L M}$ of $Q T$ dispersion caused by omission of different pairs of leads from complete 12 lead electrocardiograms. $(A)$ formula $(A)$ and $(B)$ formula $(C)$. Note that for formula $(A)$, all the relative errors are negative and the figure shows their mean values on a negative axis. Formula (C) produces both positive and negative relative errors and, for simplicity, the figure shows the absolute values of their mean values. the omission of leads II and V6 led to a mean relative error of $-0.3 \%$, but the omission of leads $\mathrm{aVL}$ and $\mathrm{V} 1$ produced a mean relative error of $-32 \cdot 7 \%$.

\section{Discussion}

INTERPRETATION OF RESULTS

The findings of this study show that there are important differences between different methods of assessment for QT dispersion with respect to compensating for missing values of QT intervals in individual electrocardiographic leads. The standard max-min formula (A) performs poorly in this respect but can be adjusted with the factor $1 / \sqrt{ } n(n=$ number of measured leads). In a population of healthy people such an adjustment removes the systematic bias introduced by missing leads of the 12 lead electrocardiogram and significantly reduces the mean relative errors due to the omission of some leads. The reasons for the success of this adjustment factor are probably statistical rather than physiological. In other words, our data suggested that the durations of QT interval in individual leads of the normal 12 lead electrocardiogram have such a statistical distribution that the $\max -\min$ (a) formula when applied to an incomplete set of leads satisfies, on average, a parabolic regression model of the number of analysed leads.

Both the unadjusted and adjusted max'-min' formula that might have been expected to be more stable because of omitting the potentially misleading extremes in the durations of QT interval were significantly worse than other approaches used in the study.

The adjustment factor $1 / \sqrt{ } n$ that was found appropriate for the $\max$ - min formula is not applicable to other methods. The $S D$ formula, which was in some aspects better than the adjusted (a) formula, may also need some adjustment because it produced a systematic bias towards lower values when applied to a smaller number of leads. The adjustment factor $1 / \sqrt{ } n$ was, however, too strong and produced much larger bias towards higher values of $\mathrm{QT}$ dispersion.

With respect to the independence of the number of analysed leads, the unadjusted $S D$ was the optimum method for the analysis of 12 lead electrocardiograms, and the weighted $S D$ (D) the optimum for the analysis of six lead chest electrocardiograms.

Despite the ability of some methods to produce minimum averaged bias when dealing with incomplete sets of electrocardiographic leads, the study also showed that the coefficients of variance of such incomplete measurements are surprisingly large. When omitting different single leads of the 12 lead electrocardiogram the $S D$ of the results of the QT dispersion, independent of the formula used, is almost three times larger than the mean of all measurements. This means that the results of all investigated formulas when applied to different subsets with only one lead missing vary substantially. With more leads 
missing, these variations become increasingly large.

The large coefficients of variance found from incomplete measurements were confirmed by the calculation of relative errors specific to individual electrocardiographic leads and to particular pairs of individual leads. Substantial differences between these relative errors were noted (differing by $>2$ orders of magnitude). This shows that although some formulas for QT dispersion can on average be adjusted for the number of measured leads, any adjustment based solely on the number of measured leads will not be successful in individual examples with missing leads.

\section{LIMITATIONS OF THE STUDY}

The main limitation of our study is the population that was investigated. The differences between QT intervals of different leads of the normal 12 lead electrocardiogram are small. This enhances the relative errors and coefficients of variance. It is possible that even the very large coefficients of variance found in this set of data will not be an obstacle to the distinction between normal and abnormally high values of QT dispersion. It is also possible that in patients with increased QT dispersion, the coefficients of variance and relative errors of QT dispersion assessed from incomplete sets of electrocardiographic leads will be different from those found in this study. Nevertheless, the results of this study do not favour the possibility of assessing QT dispersion from incomplete sets of data and such assessments might be plausible in cardiac patients only if the distribution of their durations of QT interval in 12 lead electrocardiograms is substantially and intrinsically different from that in normal people.

Also, the study did not investigate other formulas that might be used to express the differences between durations of QT interval of individual leads. Although other formulas are theoretically possible-for example skewness of the distribution of QT interval durations - they either would not correspond to the concept of QT dispersion as a measure of inhomogeneity in repolarisation, or would be very close to the formulas used in this study. Thus the selection of the methods in this study was representative.

Finally the investigation of the effects of omitting different individual leads from complete electrocardiograms did not consider the standard configuration of individual leads. It is known that in a normal electrocardiogram, the $\mathrm{T}$ wave is more likely to have a negligible voltage in some leads than in others. Practical performance of an adjustment formula should therefore be judged with respect to the likelihood of individual leads not being measurable rather than to the combinations of all incomplete electrocardiograms.

\section{PRACTICAL IMPLICATIONS}

The large values of the coefficients of variance and large differences between relative errors due to the omission of individual leads that were found in this study suggest that in normal people, the concept of adjusting QT dispersion for different numbers of electrocardiographic leads used in its assessment is at least very difficult if not impossible to fulfil. This study did not answer the question of whether this adjustment can be achieved in recordings from cardiac patients but the findings of the study throw serious doubts on such a possibility.

Thus clinical investigations that use QT dispersion assessed from different sets of electrocardiographic leads in different patients might be polluted by considerable measurement errors. The assessment of QT dispersion from different sets of electrocardiographic leads in different patients and controls seems likely to lead to mutually incomparable data.

It seems that it would be more appropriate to assess QT dispersion from some standardised sets of electrocardiographic leads. These do not necessarily need to be the complete 12 lead electrocardiograms or six lead precordial electrocardiograms. Some other combinations require further investigations-for example, the orthogonal $\mathrm{X}, \mathrm{Y}$, and $\mathrm{Z}$ leadsor the quasiorthogonal V2, I, and aVF leads, etc.

The study was supported in part by the British Heart Foundation, and by the British Council. The help of Ms Dickie with preparing the text of the manuscript is acknowledged with many thanks.

1 Day CP, McComb JM, Campbell RWF. QT dispersion: an indication of arrhythmia risk in patients with lon an indication of arrhythmia risk in pa

2 Day CP, McComb JM, Matthews J, Campbell RWF. Reduction in QT dispersion by sotalol following myocardial infarction. Eur Heart $\mathcal{f} 1991 ; 12: 423-7$.

3 Day CP, McComb JM, Campbell RWF. QT dispersion in sinus beats and ventricular extrasystoles in norma hearts. Br Heart f 1992;67:39-41.

4 Linker NJ, Colona P, Kekwick CA, Till J, Camm AJ, Ward DE Assessment of QT dispersion in symptomatic patients with congenital long QT syndromes. $A m$ f Cardiol 1992;69:634-8.

5 De Ambroggi L, Bertoni T, Locati E, Stramba-Badiale $M$ Schwartz PJ. Mapping of body surface potentials in patients with the idiopathic long QT syndrome. patients with the idiopathic

6 Rosenbaum DS, Kaplan DT, Kanai A, Jackson L, Garan H, Cohen R, Salama G. Repolarization inhomogeneities in ventricular myocardium change dynamically with abrupt cycle length shortening. Circulation 1991;84: 1333-45.

7 Lepeschkin E, Surawicz B. The measurement of the QT interval of the electrocardiogram. Circulation 1952;6: 378-88. 